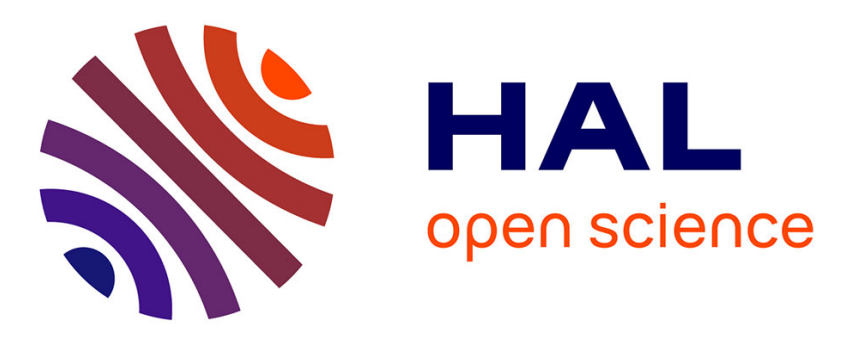

\title{
A numerical study of the topology of normally hyperbolic invariant manifolds supporting Arnold diffusion in quasi-integrable systems.
} Massimiliano Guzzo, Elena Lega, Claude Froeschle

\section{- To cite this version:}

Massimiliano Guzzo, Elena Lega, Claude Froeschle. A numerical study of the topology of normally hyperbolic invariant manifolds supporting Arnold diffusion in quasi-integrable systems.. 2009. insu$00186175 \mathrm{v} 2$

\section{HAL Id: insu-00186175}

\section{https://hal-insu.archives-ouvertes.fr/insu-00186175v2}

Preprint submitted on 27 Jan 2009

HAL is a multi-disciplinary open access archive for the deposit and dissemination of scientific research documents, whether they are published or not. The documents may come from teaching and research institutions in France or abroad, or from public or private research centers.
L'archive ouverte pluridisciplinaire HAL, est destinée au dépôt et à la diffusion de documents scientifiques de niveau recherche, publiés ou non, émanant des établissements d'enseignement et de recherche français ou étrangers, des laboratoires publics ou privés. 


\title{
A numerical study of the topology of normally hyperbolic invariant manifolds supporting Arnold diffusion in quasi-integrable systems.
}

\author{
Massimiliano Guzzo \\ Università degli Studi di Padova, \\ Dipartimento di Matematica Pura ed Applicata \\ via Trieste 63, 35121 Padova, Italy \\ Elena Lega, Claude Froeschlé \\ CNRS UMR 6202, UNSA, Observatoire de Nice, Bv. de l'Observatoire, B.P. 4229, \\ 06304 Nice cedex 4, France.
}

January 20, 2009

\begin{abstract}
We investigate numerically the stable and unstable manifolds of the hyperbolic manifolds of the phase space related to the resonances of quasi-integrable systems in the regime of validity of the Nekhoroshev and KAM theorems. Using a model of weakly interacting resonances we explain the qualitative features of these manifolds characterized by peculiar 'flower-like' structures. We detect different transitions in the topology of these manifolds related to the local rational approximations of the frequencies. We find numerically a correlation among these transitions and the speed of Arnold diffusion.
\end{abstract}

\section{Introduction}

Diffusion in generic quasi-integrable systems at small values of the perturbing parameters has been a very studied subject since the pioneering work of Arnold [1]. The proof of the existence of diffusion of orbits for generic systems satisfying the hypotheses of both KAM and Nekhoroshev theorems is still lacking, and consequently there is not a full understanding of the mechanisms which possibly produce diffusion. Since [1], many efforts have been done to relate diffusion in phase space to the topology of the so called stable and unstable manifolds of the normally hyperbolic invariant manifolds of the system, especially for the so called a priori unstable systems [5], [6], [7], [23], [24] which we studied in [3]. For quasi-integrable systems the 
situation is more complicated than for the a priori unstable ones for two reasons: first, the diffusion slows down at least exponentially with the perturbing parameter $\varepsilon$; second, the hyperbolic structures are generated by the perturbation, and hyperbolicity disappears when $\varepsilon=0$. Therefore, at any small $\varepsilon$, there is the problem of identifying the normally hyperbolic invariant manifolds of the system and to describe the properties of their asymptotic manifolds, which are clearly related to resonances.

In previous papers [16],[17],[18] we measured diffusion of the action variables in quasi-integrable systems which we identified as Arnold diffusion. To be definite, we refer to quasi-integrable systems, i.e. to Hamiltonian systems with Hamilton functions of the form:

$$
H(I, \varphi)=h(I)+\varepsilon f(I, \varphi),
$$

or to symplectic maps generated by functions of the form:

$$
S(I, \varphi)=h(I)+\varepsilon f(I, \varphi),
$$

with action-angle variables $(I, \varphi)$ defined on the open bounded domain $B \times \mathbb{T}^{n} \subseteq$ $\mathbb{R}^{n} \times \mathbb{T}^{n}$. The functions $h, f$ are such that the Hamiltonian system (1) and the map (2) satisfy the hypotheses of both KAM and Nekhoroshev theorems (for the KAM and Nekhoroshev theorems for quasi-integrable maps see [20],[21],[11]). We refer to the interval of $\varepsilon$ of application of KAM and Nekhoroshev theorem determined numerically as in [14], [15] by means of the Fast Lyapunov Indicator method ([12],[13]).

In this paper we provide for the first time a detailed description of the topology of the stable (unstable) manifolds of quasi-integrable systems (1),(2) with $n \geq 2$. Specifically, we find that when $\varepsilon \neq 0$ the topology of the stable (unstable) manifolds presents peculiar flower-like structures which we explain by using a model of weakly interacting resonances. We detect also transitions in this topology which are related to the local predominant resonances identified with the local rational approximations of the frequencies. Thank to this new description, we can show a correlation among these transitions and the Arnold diffusion.

All the numerical computations are done using the same model problem of quasiintegrable system already studied in [17], [18]. In section 2 we show that this quasiintegrable system has a resonance containing a normally hyperbolic invariant manifold $\Lambda$ which can be explicitly identified at small values of $\varepsilon \neq 0$. In section 3 we compute the stable and unstable manifolds of 2-dimensional hyperbolic invariant manifolds of the system using a numerical method, based on the Fast Lyapunov Indicator, introduced in [3]. We are able to provide a global description of the topology of the stable and unstable manifolds, and therefore to describe its peculiar flower-like structures and the correlations with the local rational approximations of the frequencies. In section 4 we provide a theoretical explanation of the flower-like 
structures. In section 5, we study the diffusion properties of the system near $\Lambda$ and we compare them with the diffusion near generic resonances. We show that the topological transitions are correlated to the diffusion properties.

\section{Normally hyperbolic invariant manifolds, resonances and a specific model of quasi-integrable system}

The notion of normally hyperbolic invariant manifolds was introduced in [4], and can be stated as follows (see, for example, [4], [9]):

Definition. Let $M$ be a $\mathcal{C}^{q}(q \geq 1)$ compact connected manifold; let $U \subseteq M$ open and let $\phi: U \rightarrow M$ be a $\mathcal{C}^{q}$ embedding; let $\Lambda$ be a sub-manifold of $M$ which is invariant by $\phi$. The map $\phi$ is said to be normally hyperbolic to $\Lambda$ ( $\Lambda$ is also said to be normally hyperbolic invariant manifold) if there exists a Riemannian structure on $M$ such that for any point $x \in \Lambda$ the tangent space $T_{x} M$ has the following splitting:

$$
T_{x} M=E^{s}(x) \oplus T_{x} \Lambda \oplus E^{u}(x)
$$

which is continuous, invariant, i.e. the linear spaces $E^{s}(x), E^{u}(x)$ are invariant by $\phi$ :

$$
D \phi E^{s}(x) \subseteq E^{s}(\phi(x)) \quad, \quad D \phi E^{u}(x) \subseteq E^{u}(\phi(x)),
$$

and there exist constants $\lambda_{1}, \lambda_{2}, \lambda_{3}, \mu_{1}, \mu_{2}, \mu_{3}$ satisfying:

$$
0<\lambda_{1} \leq \mu_{1}<\lambda_{2} \leq \mu_{2}<\lambda_{3} \leq \mu_{3} \quad, \quad \mu_{1}<1<\lambda_{3},
$$

such that:

$$
\begin{aligned}
\lambda_{1} \leq \inf _{\xi \in E^{s}(x) \backslash 0} \frac{\|D \phi(x) \xi\|}{\|\xi\|} & \leq \sup _{\xi \in E^{s}(x) \backslash 0} \frac{\|D \phi(x) \xi\|}{\|\xi\|} \leq \mu_{1} \\
\lambda_{2} \leq \inf _{\xi \in T_{x} \Lambda \backslash 0} \frac{\|D \phi(x) \xi\|}{\|\xi\|} & \leq \sup _{\xi \in T_{x} \Lambda \backslash 0} \frac{\|D \phi(x) \xi\|}{\|\xi\|} \leq \mu_{2} \\
\lambda_{3} \leq \inf _{\xi \in E^{u}(x) \backslash 0} \frac{\|D \phi(x) \xi\|}{\|\xi\|} & \leq \sup _{\xi \in E^{u}(x) \backslash 0} \frac{\|D \phi(x) \xi\|}{\|\xi\|} \leq \mu_{3}
\end{aligned} .
$$

The normally hyperbolic invariant manifolds play an important role in the studies of transport and diffusion properties of dynamical systems. In fact, they have local stable and unstable manifolds which can be used to prove the existence of diffusion in their neighborhood.

For any $x \in \Lambda$ there exist the smooth manifolds $W_{s}^{\text {loc }}(x), W_{u}^{l o c}(x)$ (see [4]) such that: $x \in W_{s}^{l o c}(x), W_{u}^{l o c}(x), T_{x} W_{s}^{l o c}(x)=E^{s}(x), T_{x} W_{u}^{l o c}(x)=E^{u}(x)$ and for any $n \geq 0$ it is:

$$
y \in W_{s}^{l o c}(x) \Rightarrow d\left(\phi^{n}(x), \phi^{n}(y)\right) \leq C\left(\mu_{1}+c\right)^{n} d(x, y)
$$




$$
y \in W_{u}^{l o c}(x) \Rightarrow d\left(\phi^{-n}(x), \phi^{-n}(y)\right) \leq C\left(\lambda_{3}-c\right)^{-n} d(x, y)
$$

with $C, c>0$ suitable constants $(c$ suitably small) and $d(\cdot, \cdot)$ denotes a distance on $M$. The manifolds $W_{s}(x), W_{s}(x)$ are then obtained by iterating the local manifolds $W_{s}^{l o c}(x), W_{u}^{l o c}(x)$ with $\phi^{-1}$ and $\phi$ respectively. The local stable and unstable manifolds of $\Lambda$ are defined by:

$$
W_{s}^{l o c}=\cup_{x \in \Lambda} W_{s}^{l o c}(x) \quad, \quad W_{u}^{l o c}=\cup_{x \in \Lambda} W_{u}^{l o c}(x),
$$

while the stable and unstable manifolds of $\Lambda$ are:

$$
W_{s}=\cup_{x \in \Lambda} W_{s}(x) \quad, \quad W_{u}=\cup_{x \in \Lambda} W_{u}(x) .
$$

While in the a priori unstable systems there exists naturally a normally hyperbolic invariant manifold whose support diffusion (see [5]), this is not the case for quasiintegrable systems. Of course, hyperbolic manifolds are related to the resonances of the system, but it can be difficult to identify them. To be definite, in this paper we refer to the discrete system used in [17], [18], [19], which is defined by the map:

$$
\phi: \quad \begin{aligned}
\mathbb{R}^{2} \times \mathbb{T}^{2} & \longrightarrow \mathbb{R}^{2} \times \mathbb{T}^{2} \\
\left(I_{1}, I_{2}, \varphi_{1}, \varphi_{2}\right) & \longmapsto\left(I_{1}^{\prime}, I_{2}^{\prime}, \varphi_{1}^{\prime}, \varphi_{2}^{\prime}\right)
\end{aligned}
$$

such that:

$$
\begin{aligned}
\varphi_{1}^{\prime} & =\varphi_{1}+I_{1} \\
\varphi_{2}^{\prime} & =\varphi_{2}+I_{2} \\
I_{1}^{\prime} & =I_{1}-\varepsilon \frac{\sin \varphi_{1}^{\prime}}{\left(\cos \varphi_{1}^{\prime}+\cos \varphi_{2}^{\prime}+c\right)^{2}} \\
I_{2}^{\prime} & =I_{2}-\varepsilon \frac{\sin \varphi_{2}^{\prime}}{\left(\cos \varphi_{1}^{\prime}+\cos \varphi_{2}^{\prime}+c\right)^{2}}
\end{aligned}
$$

where $\varepsilon$ and $c>2$ are parameters, and the symplectic structure on $\mathbb{R}^{2} \times \mathbb{T}^{2}$ is $d \varphi_{1} \wedge d I_{1}+d \varphi_{2} \wedge d I_{2}$. The map $\phi$ has the following invariant manifold:

$$
\Lambda=\left\{\left(I_{1}, \varphi_{1}, I_{2}, \varphi_{2}\right): \text { such that }\left(I_{1}, \varphi_{1}\right)=(0, \pi)\right\}
$$

for any value of the parameters. When $\varepsilon=0$ the map is integrable and $\Lambda$ is not normally hyperbolic. For $\varepsilon \neq 0$ the map is quasi-integrable, the manifold $\Lambda$ is still invariant (as well as the manifold $\left(I_{2}, \varphi_{2}\right)=(0, \pi)$ ), but one does not immediately recognize if it is normally hyperbolic and for which values of the perturbing parameter. The problem can be easily tackled by normal form theory at small values of the perturbing parameter, at least in a big measure subset of $\Lambda$ (see below). However, for given values of $\varepsilon$, which we use in our numerical experiments, we apply a 
numerical technique to test the normal hyperbolicity of the manifold $\Lambda$ (again, see below).

Normal hyperbolicity of a subset of $\Lambda$. By normal form theory it is possible to prove that if $\varepsilon$ is suitably small, then a large measure open invariant domain of $\Lambda$ is normally hyperbolic. In fact, at any point of $\Lambda$ the angle $\varphi_{1}$ is resonant, and outside the crossing with the other main resonances it is possible to conjugate with a near to the identity canonical transformation the map $\phi$ to its normal form $\phi^{\prime}$ which is a perturbation (of higher orders with respect to $\varepsilon$ ) of the map:

$$
\begin{aligned}
\varphi_{1}^{\prime} & =\varphi_{1}+I_{1} \\
\varphi_{2}^{\prime} & =\varphi_{2}+I_{2} \\
I_{1}^{\prime} & =I_{1}-\varepsilon u_{0}\left(\varphi_{1}^{\prime}\right) \\
I_{2}^{\prime} & =I_{2}
\end{aligned}
$$

where:

$$
u_{0}\left(\varphi_{1}\right)=\frac{\partial}{\partial \varphi_{1}} \frac{1}{2 \pi} \int_{0}^{2 \pi} \frac{1}{\cos \varphi_{1}+\cos \varphi_{2}+c} d \varphi_{2}=\frac{\sin \left(\varphi_{1}\right)\left(\cos \left(\varphi_{1}\right)+c\right)}{\left(\left(\cos \left(\varphi_{1}\right)+c\right)^{2}-1\right)^{\frac{3}{2}}} .
$$

The normal form $\phi^{\prime}$ can be obtained with a single step of perturbation theory (such as the ones used in [11]), and moreover the canonical conjugation, where it is defined, has $\Lambda$ as fixed invariant manifold. The map (10) is decoupled in the product of a constant twist for the variables $\varphi_{2}, I_{2}$ and a two dimensional generalized standard map for the variables $\varphi_{1}, I_{1}$ which has the fixed points $\left(\varphi_{1}, I_{1}\right)=(0,0),(\pi, 0)$. The fixed point $(0,0)$ is hyperbolic for any $c>1$ and $\varepsilon>0$ (for $\varepsilon<0$ the hyperbolic point is $(\pi, 0))$. The hyperbolic fixed point of this generalized map $\left(\varphi_{1}, I_{1}\right) \mapsto$ $\left(\varphi_{1}^{\prime}, I_{1}^{\prime}\right)$ define an hyperbolic invariant manifold for (10), which remains hyperbolic by adding small perturbations. We remark that it is possible to go beyond the normal forms constructed by one step of perturbation theory, up to a remainder which is exponentially small with respect to an inverse power of the perturbing parameter, as it is done in the proof of the Nekhoroshev theorem for maps (see [11]).

Numerical test of the normal hyperbolicity of $\Lambda$. We numerically check that the invariant manifold $\Lambda$ is normally hyperbolic for the largest value of the perturbing parameter used allover the paper: $\varepsilon=210^{-4}$. We follow the technique that we already used in paper [3]. Precisely, for a specific choice of the tangent vectors norm: $\left\|\left(\xi_{\varphi_{1}}, \xi_{\varphi_{2}}, \xi_{I_{1}}, \xi_{I_{2}}\right)\right\|^{2}=\left|\xi_{\varphi_{1}}\right|^{2}+\left|\xi_{\varphi_{2}}\right|^{2}+\left|\xi_{I_{1}}\right|^{2}+\left|\xi_{I_{2}}\right|^{2}$ we check if the invariant manifold $\Lambda$ is normally hyperbolic with respect to the map $\phi^{N}$, for some integer $N$. For each point $x$ of a grid of initial conditions on $\Lambda$ with $I_{2} \in[0,2], I_{1}=0, \varphi_{1}=\pi, \varphi_{2}=0$ we first computed the Lyapunov exponents of the map $\phi$ (up to a $N=10^{4}$ iterations) for initial tangent vectors in the tangent space $T_{x} \Lambda^{\text {ort }}$ orthogonal to $T_{x} \Lambda$, i.e. for vectors 


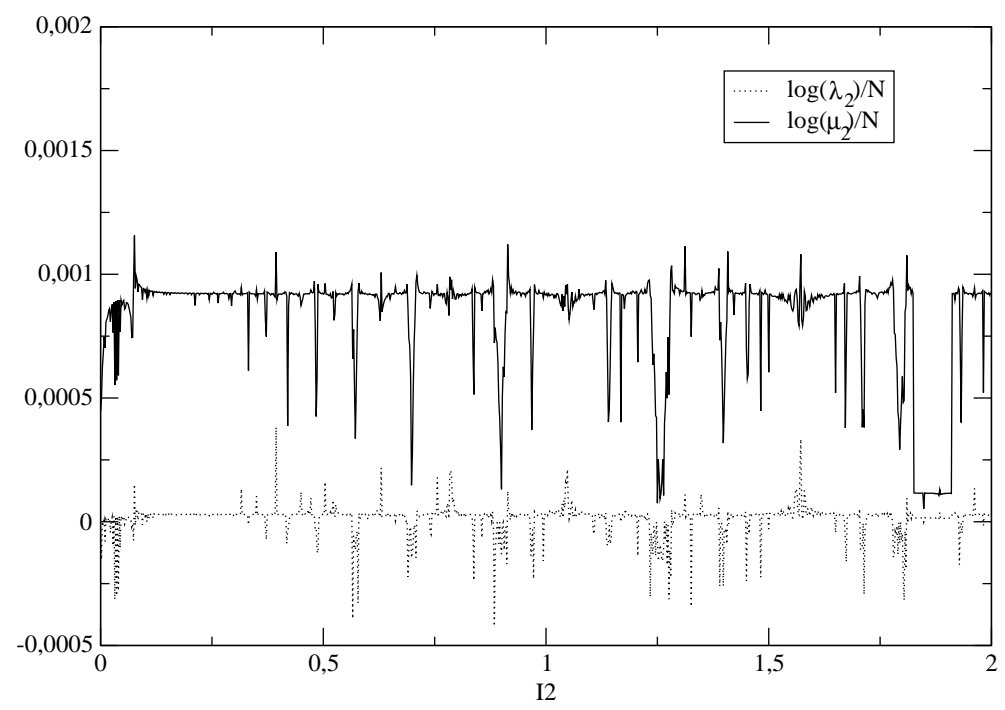

Figure 1: Numerical estimates of $\log \lambda_{2} / N$ and $\log \mu_{2} / N$, computed on a grid of 1000 initial conditions with $I_{2} \in[0,2], I_{1}=0, \varphi_{1}=\pi, \varphi_{2}=0, \varepsilon=210^{-4}, c=2.1$.

of the form $\xi=\left(\xi_{\varphi_{1}}, 0, \xi_{I_{1}}, 0\right)$. For $\varepsilon=210^{-4}$ we measured a positive Lyapunov exponent bigger than $710^{-3}$ for all the points of the grid (the negative Lyapunov exponent is smaller than $-710^{-3}$ ). This is an indication of the hyperbolic splitting of the space $T_{x} \Lambda^{\text {ort }}$ as a direct sum of a stable space $E^{s}(x)$ and an unstable space $E^{u}(x)$. The numerical algorithm for the computation of the Lyapunov characteristic exponents provides also an estimate of the constants $\lambda_{1}=\mu_{1}$ and $\lambda_{3}=\mu_{3}$ which are related to an iterate $\phi^{N}$ of $\phi$. In fact, if $N$ is sufficiently large, the quantities $1 /\left\|D \phi^{N}(x) \xi\right\|$ and $\left\|D \phi^{N}(x) \xi\right\|$ converge exponentially to $\lambda_{1}$ and $\lambda_{3}$ for almost any initial tangent vector $\xi \in T_{x} \Lambda^{\text {ort }}$. It remains to estimate the constants $\lambda_{2}, \mu_{2}$ for the map $\phi^{N}$ in the point $x$. Because in this case the growth of initial tangent vectors $\xi=\left(0, \xi_{\varphi_{2}}, 0, \xi_{I_{2}}\right) \in T_{x} \Lambda$ is not expected to be always exponential, we computed numerically the two dimensional matrix representing the restriction of $D \phi^{N}(x)$ to the space $T_{x} \Lambda$, and then we computed directly:

$$
\lambda_{2} \leq \inf _{\xi \in T_{x} \Lambda \backslash 0} \frac{\left\|D \phi^{N}(x) \xi\right\|}{\|\xi\|} \leq \sup _{\xi \in T_{x} \Lambda \backslash 0} \frac{\left\|D \phi^{N}(x) \xi\right\|}{\|\xi\|} \leq \mu_{2} .
$$

Figure 1 shows the numerical computation of $\log \lambda_{2} / N$ and $\log \mu_{2} / N$ for $N=10000$ and $\varepsilon=210^{-4}$. The comparison of the four computed quantities $\log \lambda_{1}, \log \lambda_{2}$, $\log \mu_{2}, \log \lambda_{3}$ provides numerical indication that the invariant manifold $\Lambda$ is normally hyperbolic for $\varepsilon=210^{-4}$. 
The invariant manifold $\Lambda$ is in the resonance: $I_{1}=0$. For this reason this resonance is a special one. However, the system contains a infinite set of resonances defined by:

$$
k_{1} I_{1}+k_{2} I_{2}+2 k_{3} \pi=0, k_{1}, k_{2}, k_{3} \in \mathbb{Z},
$$

and we do not find qualitative differences in the diffusion properties along $I_{1}=0$ and the other ones. Of course, using the normal forms, one can prove that all these resonances contain normally hyperbolic invariant manifolds, eventually interrupted by the double resonances. The advantage of the resonance $I_{1}=0$ is that the normally hyperbolic invariant manifold has the same expression for any $\varepsilon$ and that it is not interrupted by the double resonances. For these reasons, in this paper we study predominantly this resonant manifold, but we study also a generic one. Precisely, to compare with the results of the papers [17], [18] we study also the hyperbolic manifolds of the resonance $2 I_{2}=I_{1}$.

\section{The global topology of stable and unstable manifolds in a quasi-integrable system}

We compute the intersection among the stable and unstable manifolds of $\Lambda$ with a convenient section $S$ of the phase space, such as:

$$
S=\left\{\left(I_{1}, I_{2}, \varphi_{1}, \varphi_{2}\right):\left(\varphi_{1}, \varphi_{2}\right)=(\pi, 0)\right\}
$$

As it is explained in our previous papers [16],[17],[18], [3], such sections of phase space are very useful for the study of the topological and diffusion properties. In fact, for $\varepsilon=0$, any initial condition $\left(I_{1}, I_{2}\right)$ on the section $S$ does not return on $S$, or it returns exactly on $\left(I_{1}, I_{2}\right)$. Therefore, each invariant torus, is represented symbolically by a the initial condition of the torus belonging to $S$. If $\varepsilon \neq 0$ the actions are no more necessarily constants of motion, but if $\varepsilon$ is suitably small, the phase space if filled by a large volume of KAM tori. Anyone of these tori intersects transversely $S$ only on one point, and therefore each invariant torus is still symbolically represented by a point on $S$. The section $S$ contains possibly many points belonging to invariant tori, but these tori do not trap motions in the four dimensional phase space: there is the possibility of diffusion even for very small $\varepsilon$. This diffusion may be supported by stable and unstable manifolds, and therefore we compute the sets:

$$
S_{u}^{*}=\cup_{x \in \Lambda}\left(S \cap W_{u}(x)\right)=S \cap W_{u} \quad, \quad S_{s}^{*}=\cup_{x \in \Lambda}\left(S \cap W_{s}(x)\right)=S \cap W_{s} .
$$

The set $S_{u}^{*}$ represents the set where points with initial conditions in a neighborhood of $\Lambda$ return near $S$ following diffusion paths defined by the unstable manifolds of $\Lambda$. 
We use the Fast Lyapunov Indicator (hereafter FLI) to numerically detect the sets $S_{u}^{*}, S_{s}^{*}$, following the technique described in [3]. Let us remark that there exist many other methods of detection of stable and unstable manifolds, such as the one used in [22]. The FLI method allows us to obtain global representations of the sets $S_{u}^{*}, S_{s}^{*}$.

We recall that the FLI has been introduced in [12] and extensively investigated in [15]. As explained in [3] we compute the FLI for a grid of points of $S$ up to some given time $T$ : the points of the grid which have the highest values of the FLI are those points whose orbit approaches an hyperbolic invariant manifold within the computation time $T$, because the growth of tangent vectors is bigger near the hyperbolic manifolds. Therefore, a short-time computation of the FLI allows one to detect a neighborhood of a finite piece of the stable manifold (for the unstable manifold one repeats the computation using the inverse map). The details concerned with this method of detection of the stable and unstable manifolds are discussed in paper [3].

The first set of numerical experiments concerns the map (8) with $c=2.1$ and different values of $\varepsilon$ from $\varepsilon=610^{-7}$ up to $\varepsilon=210^{-4}$. The results of the computations are reported in figure $2{ }^{1}$ Precisely, for each value of $\varepsilon$ we computed the FLI on a grid of $1000 \times 1000$ points of $S$ regularly spaced in $I_{1}$ chosen in a small neighborhood of $I_{1}=0$, while the action $I_{2}$ is in suitable neighborhoods of $I_{2}=0.324$. The higher values of the FLI are reported in light gray in the pictures.

The light gray line at $I_{1}=0$ represents the normally hyperbolic invariant manifold $\Lambda$. Around $\Lambda$ we observe also the presence of peculiar structures related to the dominant resonances, that we will explain in section 4. Here, we remark that these structures are made by different petals centered at crossings of $\Lambda$ with other resonances. Moreover, we observe that at different values of $\varepsilon$ the dominant structures which characterize the topology of the stable manifolds in a specific domain of the action space are different, according to the local dominant resonances. We are specifically interested in identifying the structures which mainly characterize the topology of $S_{s}^{*}, S_{u}^{*}$ in a neighborhood of $\left(I_{1}, I_{2}\right)=(0,0.324)$, which is the point of $\Lambda$ around which we computed the diffusion coefficient for many different values of $\varepsilon$ (see figure 9). Our aim is to compare the topology of $S_{s}^{*}, S_{u}^{*}$ with the diffusion coefficient for the different values of $\varepsilon$. Specifically, for any given value of $\varepsilon$, we identify the center of the flower-like structure which mainly characterizes the topology of $S_{s}^{*}, S_{u}^{*}$ in a neighborhood of $\left(I_{1}, I_{2}\right)=(0,0.324)$.

\footnotetext{
${ }^{1}$ The color version of all figures can be found on the electronic version of the paper so that light gray corresponds there to yellow and darker gray corresponds there to red-violet. To better appreciate the topology we uploaded high resolution pictures available also at http://www.obsnice.fr/elena/flowers. In the final version of the paper the pictures should be available on-line as supplemental material.
} 
To help us in the identification of the dominating structures we remark that, in the integrable approximation, the resonances intersect the invariant manifold $\Lambda$ in:

$$
\left(I_{1}, I_{2}\right)=\left(0, \frac{k_{1}}{k_{2}} 2 \pi\right)
$$

with $k_{1}, k_{2} \in \mathbb{Z}$. Therefore, in the neighborhood of $\left(I_{1}, I_{2}\right)=(0,0.324)$, we can find flower-like structures centered around the points (13), with $k_{1}, k_{2}$ defined by the main approximants of 0.324 . The first points of the approximating sequence are:

$$
I_{2}=0 \quad, \quad \frac{2}{19} \pi \sim 0.3306 \quad, \quad \frac{4}{39} \pi \sim 0.3221, \frac{3}{29} \pi \sim 0.3249 \quad \ldots
$$

We found the four different cases:

i) for $10^{-4} \leq \varepsilon \leq 310^{-4}$ the dominant flower-like structure around the point $\left(I_{1}, I_{2}\right)=(0,0.324)$ is centered on $I_{2}=0$, which is the first point of the approximating sequence (14). In figure 2, top left panel, we report the computation of the FLI for $\varepsilon=210^{-4}$ and $T=600$ iterations. One can appreciate that the petals around the point $\left(I_{1}, I_{2}\right)=(0,0.324)$ are centered on $\left(I_{1}, I_{2}\right)=(0,0)$.

ii) For $710^{-6} \leq \varepsilon<10^{-4}$ we found that the dominant flower-like structure is centered on $I_{2}=2 \pi / 19$, which is the second point of the approximating sequence (14). In figure 2, top right panel, we report the computation of the FLI for $\varepsilon=310^{-5}$ and $T=2000$ iterations.

iii) For $610^{-7} \leq \varepsilon<710^{-6}$ we found that the dominant flower-like structure is centered on $\left(I_{1}, I_{2}\right)=(0,4 \pi / 39)$, which is the third point of the approximating sequence (14). In figure 2 we report the computation of the FLI for $\varepsilon=$ $5.610^{-6}$ and $T=700$ iterations. (bottom left panel).

iv) For $\varepsilon<610^{-7}$ we observe that the dominant flower-like structure is centered on $\left(I_{1}, I_{2}\right)=(0,3 \pi / 29)$, which is the fourth point of the approximating sequence (14). In figure 2 we report the computation of the FLI for $\varepsilon=610^{-7}$ and $T=30000$ iterations (bottom right panel). The point $\left(I_{1}, I_{2}\right)=(0,0.324)$ appears at the junction between the petals centered on the third and on the fourth point of the approximating sequence (14).

We anticipate that in section 5 we will show that the intervals of $\varepsilon$ corresponding to the different dominating topological structures are correlated to different diffusion properties.

A second set of numerical experiments concerns the map (8) with $c=4$ and a neighborhood of the resonance $I_{1}=2 I_{2}$. The reason to study this case is twofold: 
on the one hand this resonance is generic, in the sense that it is not trivially related to a normally hyperbolic invariant manifold such as the resonance $I_{1}=0$; on the other hand it is the resonance which we studied in the papers [17],[18]. In figure 3 we report the computation of the FLI for values of $\varepsilon$ ranging from 0.04 up to 0.4. The coordinates of the pictures are $x=I_{2}-I_{1} / 2, y=I_{2}+2 I_{1}$, so that the resonance is almost vertical. In all the pictures we appreciate the presence of an hyperbolic structure which plays the role of the normally hyperbolic invariant manifold $\Lambda$. We will refer to this structure as to the hyperbolic set of the resonance. Around this hyperbolic set we observe also the presence of the flower-like structures (which will be explained in section 4) related to the dominant resonances. As for the previous case, at different values of $\varepsilon$ the dominant flower-like structures which characterize the topology of the stable manifold are different, according to the local dominant resonances. In fact, for $\varepsilon=0.04$ (top left panel of figure 3) we detect only one flowerlike structure centered around a resonance which cross the hyperbolic invariant set in the middle of the figure. For $\varepsilon=0.1$ (top right panel of figure 3 ) in the same region of the phase-space we observe also the petals of flower-like structures which do not belong to the central one, but are centered around resonances which are outside the upper and the lower sides of the picture. For $\varepsilon=0.22$ (bottom left panel of figure 3 ) the upper and lower structures have become dominant with respect to the central one, which is no more evident in this picture. For $\varepsilon=0.4$ the upper and lower structures are overlapping in correspondence of the central resonance. It is clear that these transitions in the topology of the stable manifold are related to the local dominant resonances, which are different at different values of $\varepsilon$. We observe that also in this case the transitions in the topology of the stable manifold are related to the changes of the diffusion properties around the resonance.

\section{Flower-like structure of the stable manifolds in the case of two weakly interacting resonances}

In the previous section we detected the topology of the stable (unstable) manifolds of the normally hyperbolic invariant manifold $\Lambda$, and we found that they are characterized by the presence at any scale of peculiar structures, made of many petals, which we called "flower-like".

Now, we explain the flower-like structures of the stable and unstable manifolds of resonances in a model with two weakly interacting resonances of the same amplitude. Precisely, we consider the system with Hamilton function:

$$
H\left(I_{1}, I_{2}, \varphi_{1}, \varphi_{2}\right)=\frac{I_{1}^{2}}{2}+\frac{I_{2}^{2}}{2}-a_{1} \cos \left(\varphi_{1}\right)-a_{2} \cos \left(\varphi_{2}\right)-\varepsilon \cos \left(\varphi_{1}\right) \cos \left(\varphi_{2}\right),
$$


where $a_{1}, a_{2}$ are constants of order 1 and $\varepsilon$ is a small parameter. Hyperbolicity is present for both couples of variables $\left(I_{1}, \varphi_{1}\right),\left(I_{2}, \varphi_{2}\right)$. The perturbation introduces interaction among all the variables. We remark that the normal form of a generic $n$ degrees of freedom Hamiltonian system $H(I, \varphi)=h(I)+\eta f(I, \varphi)$ near a double resonance takes the form, in suitable scaled action-angle variables $J, \psi$ [10],

$$
\mathcal{H}(J, \psi)=k(J)+g\left(\psi_{1}, \psi_{2}\right)+\sqrt{\eta} u\left(J, \psi_{1}, \psi_{2} ; \eta\right)+\exp -\left(\frac{\eta_{0}}{\eta}\right)^{\frac{1}{2 n}} v(J, \psi ; \eta)
$$

In the frequent case of double resonances characterized by two dominating harmonics the function $g$ can be written in the form:

$$
g=g_{1}\left(\psi_{1}\right)+g_{2}\left(\psi_{2}\right)+\varepsilon g_{12}\left(\psi_{1}, \psi_{2}\right),
$$

so that (16) has the form of a small perturbation of an Hamiltonian similar to (15).

Returning to Hamiltonian (15), we remark that it has the normally hyperbolic invariant manifold:

$$
\Lambda=\left\{\left(I_{1}, I_{2}, \varphi_{1}, \varphi_{2}\right): \varphi_{1}=\pi, I_{1}=0\right\}
$$

(as well as other ones) and we want to describe its asymptotic manifolds. In figure 4, left panel, we report the computation of the FLI on the section $S$ defined by (12): we can appreciate the presence of a peculiar flower-like structure. Then, we repeat the computation for a system which is an approximation of (15). Precisely, we first approximate the equation for the variable $\varphi_{1}$ as follows:

$$
\ddot{\varphi}_{1}=-\sin \left(\varphi_{1}\right)\left(a_{1}+\varepsilon \cos \left(\varphi_{2}^{0}(t)\right)\right)+\mathcal{O}\left(\varepsilon^{2}\right)
$$

where $\varphi_{2}^{0}(t)$ is the order-0 solution for $\varphi_{2}$, i.e. $\ddot{\varphi}_{2}^{0}=-a_{2} \sin \left(\varphi_{2}^{0}\right)$, and we further approximate the equation by neglecting the $\mathcal{O}\left(\varepsilon^{2}\right)$ term. Therefore, the approximated system can be written in the form:

$$
\begin{aligned}
\dot{\varphi}_{1} & =I_{1} \\
\dot{\varphi}_{2}^{0} & =I_{2}^{0} \\
\dot{I}_{1} & =-\sin \left(\varphi_{1}\right)\left(a_{1}+\varepsilon \cos \left(\varphi_{2}^{0}\right)\right) \\
\dot{I}_{2}^{0} & =-a_{2} \sin \left(\varphi_{2}^{0}\right),
\end{aligned}
$$

and we describe the topology of the asymptotic manifolds of its hyperbolic invariant manifold:

$$
\Lambda_{0}=\left\{\left(I_{1}, I_{2}^{0}, \varphi_{1}, \varphi_{2}^{0}\right): \varphi_{1}=\pi, I_{1}=0\right\} .
$$


In figure 4, right panel, we report the computation of the FLI on the section $S$ for the approximated system (17). From the comparison between the two panels of figure 4 we can appreciate that the flower-like structures of the complete system are accurately reproduced also in the approximated one, except very close to the resonance $I_{2}=0$, where the approximation (17) is not accurate. Using (17), we are able to explain that the intersection of the stable manifold of $\Lambda_{0}$ with the section $S$ is made of the "petals" structures represented in figure 4.

For any initial condition $\varphi_{2}^{0}(0)=\pi, \dot{\varphi}_{2}^{0}(0)=I_{2}^{0}(0)$ the solution $\varphi_{2}^{0}(t)$ is periodic with some period $T\left(I_{2}^{0}(0)\right)$. For any fixed $I_{2}^{0}(0)$, we consider the Poincaré section $\phi^{T}$ at time $T$ of the Hamiltonian system:

$$
H=\frac{I_{1}^{2}}{2}-a_{1} \cos \left(\varphi_{1}\right)-\varepsilon \cos \left(\varphi_{1}\right) \cos \left(\varphi_{2}^{0}(t)\right) .
$$

The stable and unstable manifolds of the fixed point $x=\left(\varphi_{1}, I_{1}\right)=(\pi, 0)$ for the map $\phi^{T}$ are characterized by the well known structure of the homoclinic tangle of 2-dimensional maps. We consider $W_{s}(x)$ and its first four intersections with the axis $\varphi_{1}=\pi$, which are marked with stars in figure 5 . By changing $I_{2}^{0}(0)$, the intersection points of the unstable manifold with the axis $\varphi_{1}=\pi$ change as well, describing arcs on the section $S$. When a lobe of the homoclinic tangle becomes tangent to the axis $\varphi_{1}=\pi$, two arcs of intersection points on the section $S$ are joint together, and they constitute the upper part of a petal of the flower-like structure. By moving $I_{2}^{0}(0)$ towards zero, the value of $I_{2}^{0}$ of the intersection points goes to zero as well, because for $I_{2}^{0}(0)=0$ the function $\varphi_{2}^{0}(t)$ is constant with respect to time, and therefore the system is integrable and the unstable manifold has not lobes. Therefore, the generation of a petal of the flower-like structure is the following: starting from the exact resonance we find the basis of the petal; increasing $I_{2}(0)$, i.e. going away from the resonance, a lobe intersects the section producing the two arcs of the petal, which are joint together for the value of $I_{2}(0)$ at which that lobe is tangent to the section. We remark that the petals generated this way have their basis at the exact resonance, but, as one can understand from picture 5 (top panel), they belong to the stable manifold of the single resonance $I_{1}=0$. Therefore, though the flower-like structures are centered on the double resonances, many of their petals belong to the stable manifolds of the single resonances. In figure 6 we report the computation of the FLI for the map (8). The parameters of the map $(\varepsilon=0.0001, c=2.1)$ and the resolution of the figure allow to appreciate the presence of many flower-like structures centered around the resonance crossings, and the 'long petals' which are part of the stable manifolds of the single resonances. For this value of $\varepsilon$, all these hyperbolic structures coexist with a large measure set of invariant tori, as in the regime of validity of KAM theorem. 


\section{Correlation between Arnold diffusion and the topol- ogy of the stable manifolds}

In this section we study numerically the diffusion properties of the map $\phi$ near the invariant manifold $\Lambda$. We remark that the dynamics of $\phi$ restricted to $\Lambda$ is represented by the 2 -dimensional standard map:

$$
\varphi_{2}^{\prime}=\varphi_{2}+I_{2} \quad, \quad I_{2}^{\prime}=I_{2}-\varepsilon \frac{\sin \varphi_{2}^{\prime}}{\left(\cos \varphi_{2}^{\prime}+c-1\right)^{2}},
$$

whose phase portraits are characterized, at small values of $\varepsilon$, by KAM curves, resonant librations and chaotic motions. In particular, because of the existence of KAM curves, the map $\phi$ restricted to $\Lambda$ has no diffusion. By analyzing the phase portraits of the map for $c=2.1$ we find (see figure 7 ) that, approximately for $\varepsilon<\varepsilon_{0} \sim 0.002$, the map has invariant KAM tori which are complete barriers to chaotic diffusion. But, even if for $0 \leq \varepsilon<\varepsilon_{0}$ there is not global diffusion for orbits with initial conditions exactly on $\Lambda$, possibility of diffusion exists for orbits with initial conditions in small neighborhoods of $\Lambda$. Using the method described in [14], we numerically find indication that the KAM and Nekhoroshev theorems apply at least for $0 \leq \varepsilon<\varepsilon_{1}=310^{-4}$. We remark that the critical values $\varepsilon_{0}$, $\varepsilon_{1}$ satisfy: $\varepsilon_{1}<\varepsilon_{0}$.

We measure the properties of diffusion in a neighborhood of $\Lambda$ by using the techniques described in [16], [17], [18], [19]. First, we observe that generic individual orbits in a neighborhood of the invariant manifold indeed spread in the $I_{2}$ direction, as it is shown in figure 8, left panel. Second, we show that the orbits spread with statistical regularity. We say that a set of $N$ orbits diffuse in the phase space with regular statistics if the average evolution of the squared distance of the actions from their initial value grows linearly with time; i.e. there exists a constant $D>0$, which we call diffusion coefficient, such that:

$$
\frac{\sum_{j=1}^{N}\left\|I^{j}(t)-I^{j}(0)\right\|^{2}}{N} \propto D t
$$

where $I^{j}(t)$ denote the time evolution of the actions for the $N$ orbits. We require that the linear law (20) is verified by means of a $\chi$-square fit with correlation coefficient larger than 0.9. The diffusion coefficient related to the individual action $I_{2}$ will be here considered, and it is defined as the constant $D_{2}$ which best fits the evolution law:

$$
\frac{\sum_{j=1}^{N}\left|I_{2}^{j}(t)-I_{2}^{j}(0)\right|^{2}}{N} \propto D_{2} t
$$


In [16], [17], [18], [19] we found sets of orbits diffusing with regular statistics, with diffusion coefficient $D$ decreasing with $\varepsilon$ faster than power laws, possibly exponentially, in agreement with Nekhoroshev theorem. Therefore, any eventual diffusion of the actions at small values of $\varepsilon$ occurs with very small diffusion coefficient. In this situation, as it is shown in [16], [17],[18], the numerical fit of diffusion coefficients can be affected by very large errors. The most important contribution to these errors is provided by the correlations in the evolution of $\left(I_{2}(t)-I_{2}(0)\right)^{2}$ with the values of the angles $\varphi_{1}(t), \varphi_{2}(t)$. In [16] it was shown that these correlations are so large that they can mask the linear growth characteristic of the diffusion process. In [16], $[17],[18]$ we solved the problem by computing the diffusion coefficient through two dimensional sections of the phase-space, such as:

$$
S=\left\{\left(I_{1}, \varphi_{1}, I_{2}, \varphi_{2}\right) \text { such that }: \varphi_{1}=\pi, \varphi_{2}=0\right\}
$$

The computation of the diffusion coefficient through the section $S$ requires some technical recipes which are described at the end of the section. Here, we report the results of computation of $D_{2}$ for many different values of $\varepsilon$, see figure 9 . In agreement with the results of [16],[17], we find that $D_{2}(\varepsilon)$ can not be fitted to a single power law in $\varepsilon$, while we fitted the data to three different power laws $D_{2}(\varepsilon) \simeq \varepsilon^{m}$ with respectively $m 1=5.4, m 2=9.8$ and $m 3=24.9$ in different intervals of $\varepsilon$. Let us remark that this kind of dependence is consistent with a regime of validity of the Nekhoroshev theorem for the symplectic maps (see for example [11]). A semianalytical study about the connection between the remainders of the resonant normal forms and Arnold diffusion for the systems studied in [16],[17] is published in [8].

The intervals of $\varepsilon$ corresponding to the different slopes $m 1, m 2, m 3$ reported in figure (9) correspond to the intervals characterized by different dominating topological structures: $m 1$ characterizes the interval (i) reported in section $4, m 2$ characterizes the interval (ii) and $m 3$ characterizes the interval (iii). Therefore, there is a correlation between the slopes $m 1, m 2, m 3$ and the points of the approximating sequence (14); i.e. the resonances whose flower-like structures dominate the topology of $S_{s}^{*}, S_{u}^{*}$ in a neighborhood of $\left(I_{1}, I_{2}\right)=(0,0.324)$.

These correlations depend on the fact that the local dominant resonances are different at different values of $\varepsilon$. This fact can be related to the construction of the resonant normal forms in the proof of the Nekhoroshev theorem, where different domains of the action space are related to different resonances according to the value of $\varepsilon$.

We observe similar correlations for the resonance $I_{1}=2 I_{2}$, which we considered in section 4 as well. The diffusion coefficient along this resonance is reported in figure 6 of [18]: $\varepsilon=0.04$ is in the interval characterized by $D(\varepsilon) \sim \varepsilon^{m 3}$, with $m 3 \sim 13.3$; $\varepsilon=0.1$ is in the transition between the law $D(\varepsilon) \sim \varepsilon^{m 3}$ and the law $D(\varepsilon) \sim \varepsilon^{m 2}$, 
with $m 2 \sim 8.5 ; \varepsilon=0.22$ is in the interval characterized by $D(\varepsilon) \sim \varepsilon^{m 2}$ and $\varepsilon=0.4$ is in the interval characterized by $D(\varepsilon) \sim \varepsilon^{m 1}$, with $m 1=4.2$.

A very similar correlation among the rational approximations of a forcing frequency and the slopes characterizing the exponentially small change of an adiabatic invariant were measured also in [2]. Also in that case, while a Nekhoroshev like theory provided an exponential upper bound on the variation of the adiabatic invariant, the numerical computation showed that the real change of the adiabatic invariant corresponded to a sequence of local power laws $\varepsilon^{m}$ which is consistent (but not identical) to the exponential upper bound. Moreover, the exponents $m$ were related to the rational approximations of the forcing frequency.

Numerical measures of $D_{2}$. In figure 9 we reported the computation of the diffusion coefficient $D_{2}$ through $S$. Practically, we have considered in the fit of (21) only the values $I_{2}^{j}(t)$ such that:

$$
\left|\varphi_{1}^{j}(t)-\pi\right| \leq \sigma_{1} \quad, \quad\left|\varphi_{2}^{j}(t)\right| \leq \sigma_{2}
$$

with $\sigma_{1}, \sigma_{2}$ sufficiently small so that the correlations in $I_{2}$ due to the variations of the angles are drastically reduced. Because not all the orbits satisfy condition (23) at the same time $t$, we choose a finite fraction $\delta t$ of the integration time and we compute the quantity:

$$
\bar{d}(n \delta t)=\frac{1}{M_{n}} \sum_{j:\left(\left|\varphi_{1}^{j}(t)-\pi\right| \leq \sigma_{1},\left|\varphi_{2}^{j}(t)\right| \leq \sigma_{2}\right)}\left(I_{2}^{j}(t)-I_{2}^{j}(0)\right)^{2} \quad n \in \mathbb{N},
$$

where $M_{n}$ is the number of points satisfying (23) for $t \in[(n-1) \delta t, n \delta t)$. The diffusion coefficient $D_{2}$ is estimated as the slope of the best linear fit of the quantity $\bar{d}(n \delta t)$.

We measured the diffusion coefficient $D_{2}$ for $c=2.1$ and for many different values of $\varepsilon$. The results were reported in figure (9). Depending on the value of $\varepsilon$, we found convenient to use data sets of $N=100$ or $N=1000$ orbits and total integration times ranging from $t=10^{8}$ up to $t=10^{10}$. The initial conditions of each data set are: $I_{2}=0.324, \varphi_{1}=\pi, \varphi_{2}=0$ for all the orbits of the data set, while the $N$ different orbits correspond to $N$ different initial conditions for the variable $I_{1} \in[-d, d]$, with $d$ ranging from $10^{-5}$ up to $10^{-2}$, depending on the value of $\varepsilon$. The diffusion coefficient for $\varepsilon=5.2510^{-6}$ is obtained on $10^{10}$ iterations using $\sigma_{j}=10^{-3}, j=1,2$. For $\varepsilon<5.2510^{-6}$ the diffusion coefficient is so small that we need to adapt the previous method. First, we fix $\sigma_{1}=10^{-6}, \sigma_{2}=10^{-4}$ and consider $N=1000$ orbits with initial conditions $I_{2}=0.324, \varphi_{1}=\pi, \varphi_{2}=0$ and $I_{1} \in\left[-10^{-11}, 10^{-11}\right]$. Second, we remark that the points returning on the double section show an approximate linear 
correlation between $I_{2}$ and $\varphi_{2}$ (figure 10,left for $t=610^{5}$ ), and we computed the best linear fit $I_{2}=m \varphi_{2}+q$ (plotted in figure 10,left). Finally, we estimate the diffusion coefficient by fitting the quantity:

$$
\tilde{d}(n \Delta t)=\frac{1}{M_{n}} \sum_{j:\left(\left|\varphi_{1}^{j}(t)-\pi\right| \leq \sigma_{1},\left|\varphi_{2}^{j}(t)\right| \leq \sigma_{2}\right)}\left(I_{2}^{j}(t)-\tilde{I}_{2}^{j}(0)\right)^{2}
$$

where $\tilde{I}_{2}^{j}(0)=m \varphi_{2}^{j}(t)+q$. The diffusion coefficient computed for $\varepsilon=5.2510^{-6}$ using equation (24) with $t=10^{10}, N=1000, \sigma_{j}=10^{-3}, j=1,2$ turns out to be the same when computed using equation (25) with $t=10^{9}, N=1000, \sigma_{1}=10^{-6}$, $\sigma_{2}=10^{-4}$. We could then still decrease $\varepsilon$ and compute $D_{2}$ with the same previous set of parameters down to $\varepsilon=3.510^{-6}$.

Acknowledgments. M. Guzzo has been supported by the project CPDA063945/06 of the University of Padova.

\section{References}

[1] Arnold V.I.: Instability of dynamical systems with several degrees of freedom. Sov. Math. Dokl., 6, 581-585, 1964.

[2] Benettin G. and Fassò F.: From Hamiltonian perturbation theory to symplectic integrators and back. Proceedings of the NSF/CBMS Regional Conference on Numerical Analysis of Hamiltonian Differential Equations (Golden, CO, 1997). Appl. Numer. Math. 29, 1, 73-87, 1999.

[3] Guzzo M., Lega E. and Froeschlé C.: A numerical study of the topology of hyperbolic manifolds supporting Arnold diffusion in a priori unstable systems. Preprint, available at http://hal-insu.archives-ouvertes.fr/insu-00186172/fr/.

[4] Hirsch M.W., Pugh C.C. and Shub M.: Invariant Manifolds. Lecture Notes in Mathematics, Vol. 583. Springer-Verlag, Berlin-New York, 1977.

[5] Chierchia L. and Gallavotti G.: Drift and diffusion in phase space. Ann. Inst. H.Poincaré, Vol. 60, 1-144, 1994.

[6] Chierchia L. and Valdinoci E.: A note on the construction of Hamiltonian trajectories along heteroclinic chains. Forum Math., Vol. 12, 247-255, 2000. 
[7] Delshams A., de la Llave R. and Seara T. M.: A geometric mechanism for diffusion in Hamiltonian systems overcoming the large gap problem: heuristics and rigorous verification on a model. Mem. Amer. Math. Soc. 179, no. 844, 2006.

[8] Efthymiopoulos C.: On the connection between the Nekhoroshev theorem and Arnold Diffusion. Celestial Mechanics and Dynamical Astronomy, Vol. 102, n. 1-3, 49-68, 2008.

[9] Hasselblatt B. and Pesin Y.: Partially hyperbolic dynamical systems. Handbook of dynamical systems. Vol. 1B, 1-55, Elsevier B. V., Amsterdam, 2006.

[10] Benettin, G. and Gallavotti G.: Stability of motions near resonances in quasiintegrable Hamiltonian systems. J. Stat. Phys., vol. 44, 293-338, 1986.

[11] Guzzo M.: A direct proof of the Nekhoroshev theorem for nearly integrable symplectic maps. Annales Henry Poincaré, vol. 5, n. 6, 1013-1039, 2004.

[12] Froeschlé C., Lega E. and Gonczi R.: Fast Lyapunov indicators. application to asteroidal motion. Celest. Mech. and Dynam. Astron., Vol. 67, 41-62, 1997.

[13] Froeschlé C., Gonczi R. and Lega E.: The fast Lyapunov indicator: a simple tool to detect weak chaos. Application to the structure of the main asteroidal belt. Planetary and space science, Vol. 45, 881-886, 1997.

[14] Froeschlé C., Guzzo M. and Lega E.: "Graphical Evolution of the Arnold Web: From Order to Chaos". Science, Volume 289, n. 5487, 2000.

[15] Guzzo M., Lega E. and Froeschlé C.: On the numerical detection of the effective stability of chaotic motions in quasi-integrable systems. Physica D, Volume 163, Issues 1-2, 1-25, 2002.

[16] Lega E., Guzzo M. and Froeschlé C.: "Detection of Arnold diffusion in Hamiltonian systems". Physica D, vol. 182, p. 179-187, 2003.

[17] Guzzo M., Lega E. and Froeschlé C.: "First Numerical Evidence of Arnold diffusion in quasi-integrable systems". DCDS B, vol. 5, n. 3, 2005.

[18] Froeschlé C., Guzzo M. and Lega E.: "Local and global diffusion along resonant lines in discrete quasi-integrable dynamical systems", Celestial Mechanics and Dynamical Astronomy, vol. 92, n. 1-3, 243-255, 2005.

[19] Guzzo M., Lega E. and Froeschlé C.: Diffusion and stability in perturbed non-convex integrable systems. Nonlinearity, 19, p 1049-1067, 2006. 
[20] Kuksin, S. B.: On the inclusion of an almost integrable analytic symplectomorphism into a Hamiltonian flow. Russian journal of Mathematical Physics, 1, 2, 191-207 (1993).

[21] Kuksin, S. and Pöschel, J.: On the inclusion of analytic symplectic maps in analytic Hamiltonian flows and its applications. Seminar on Dynamical Systems (St. Petersburg, 1991), 96-116, Progr. Nonlinear Differential Equations Appl., 12, Birkhäuser, Basel, 1994.

[22] Simo C. and Valls C.: A formal approximation of the splitting of separatrices in the classical Arnold's example of diffusion with two equal parameters. Nonlinearity 14, no. 6, 1707-1760, 2001.

[23] Treschev D.: Trajectories in a neighbourhood of asymptotic surfaces of a priori unstable Hamiltonian systems. Nonlinearity 15 2033-2052, 2002.

[24] Treschev D.: Evolution of slow variables in a priori unstable Hamiltonian systems. Nonlinearity 17 1803-1841, 2004. 

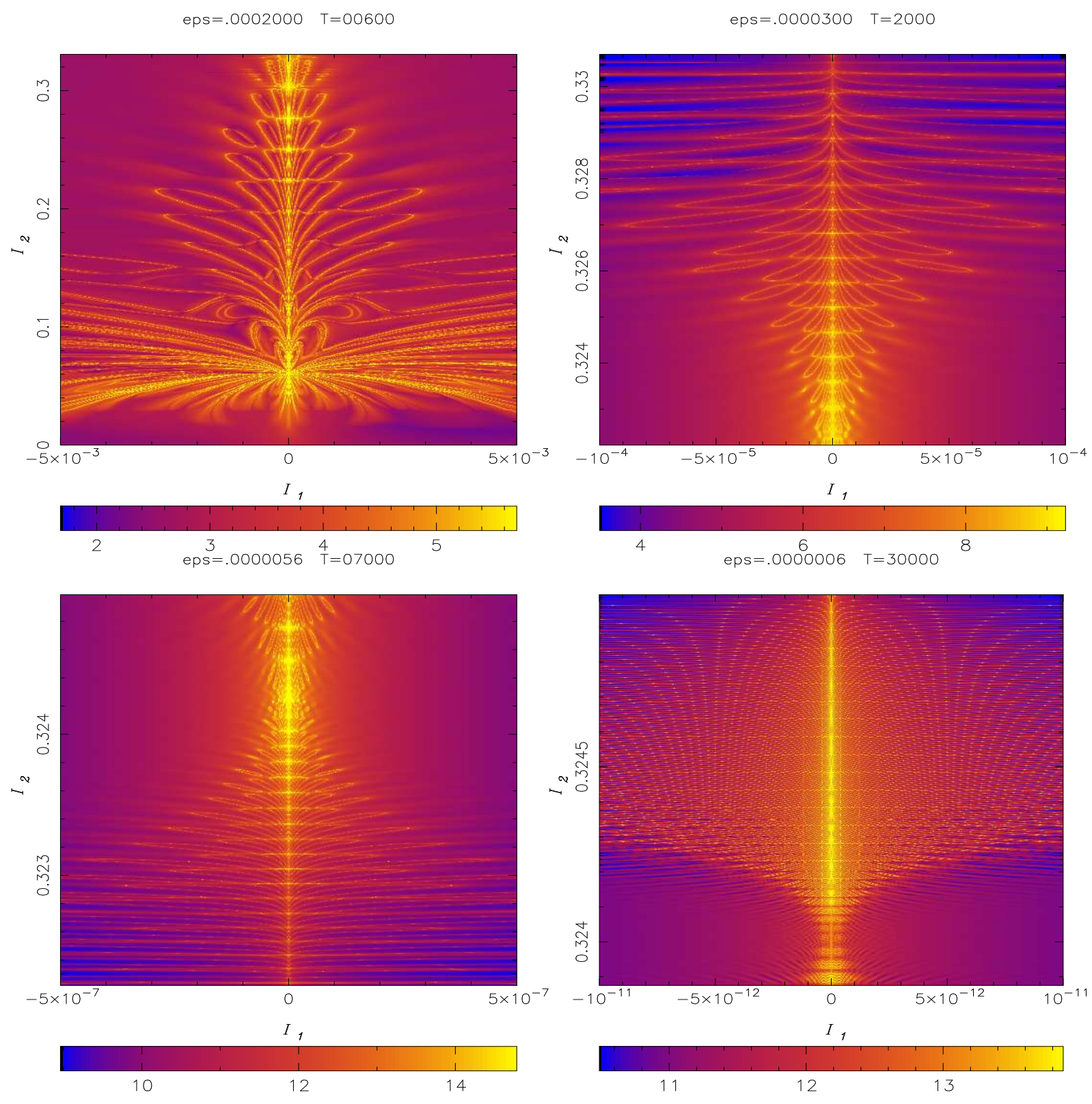

Figure 2: Computation of the FLI on the section $S$ for $c=2.1$ and $\varepsilon=210^{-4}, 310^{-5}$ (on the top) and $\varepsilon=5.610^{-6}, 610^{-7}$ (on the bottom). 

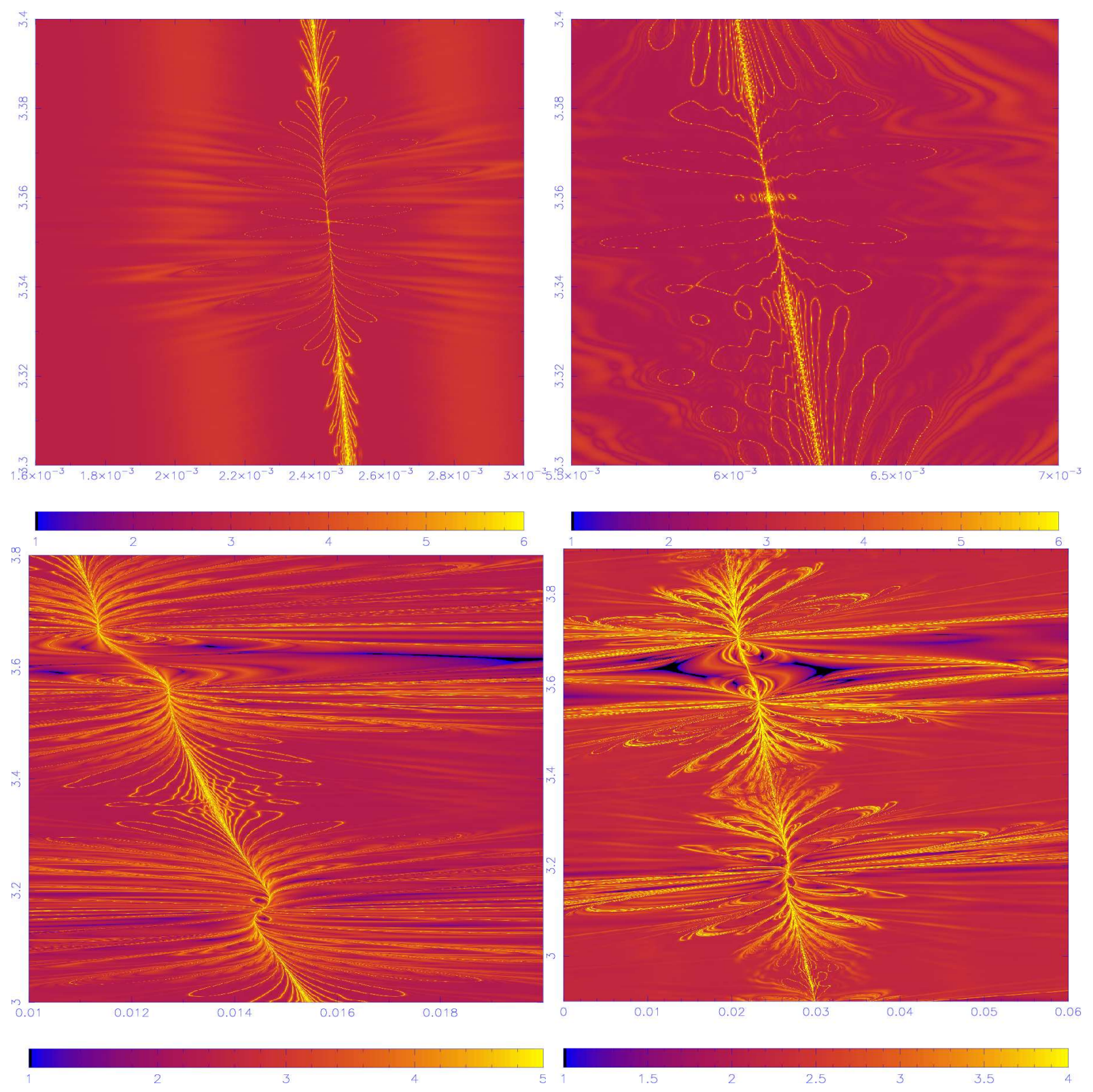

Figure 3: Computation of the FLI for $c=4$ and $\varepsilon=0.04,0.1$ (on the top) and $\varepsilon=0.22,0.4$ (on the bottom). The coordinates of the pictures are $x=I_{2}-I_{1} / 2$, $y=I_{2}+2 I_{1}$, so that the resonance is almost vertical. 

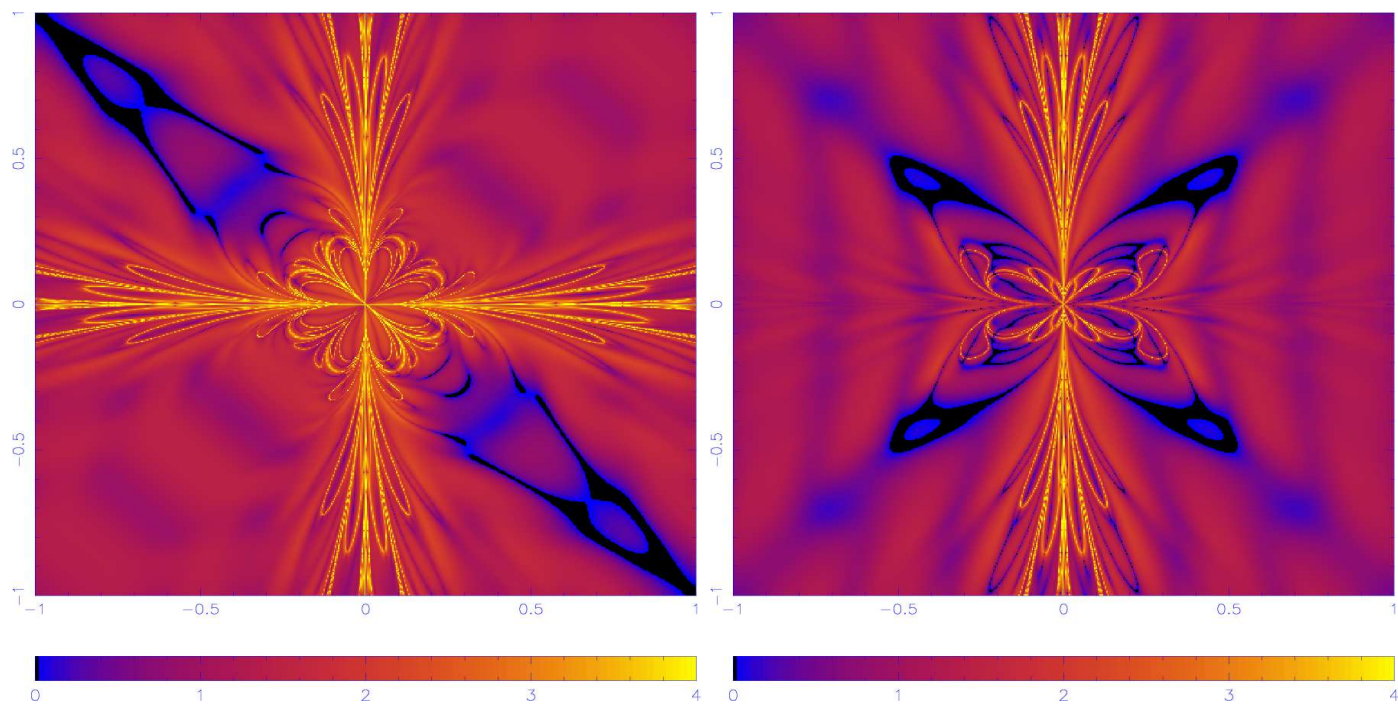

Figure 4: Left panel: asymptotic manifolds of the hyperbolic invariant manifolds of Hamiltonian systems (15), for $a_{1}=a_{2}=1$ and $\varepsilon=0.01$. Right panel: asymptotic manifolds of the hyperbolic invariant manifolds of the approximated system (17), for $a_{1}=a_{2}=1$ and $\varepsilon=0.01$. 

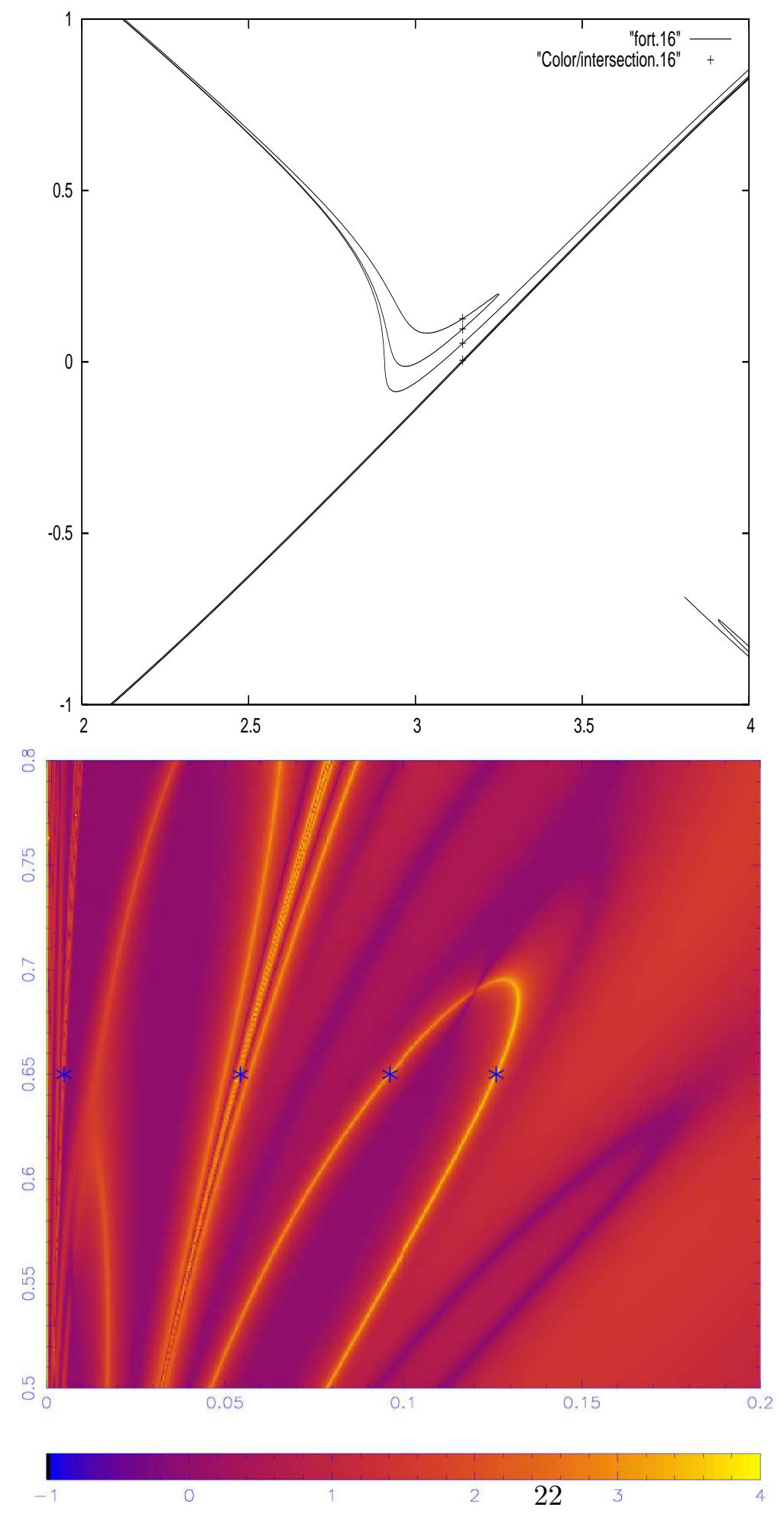

Figure 5: Top panel: zoom of the unstable manifold around the hyperbolic fixed point obtained for $a_{1}=a_{2}=1, \varepsilon=0.01, I_{2}(0)=0.65$. The stars represent some intersection points of the manifold with the section $\varphi_{1}=\pi$, which are then reproduced also on the bottom panel. Bottom panel: Unstable manifold of $\Lambda$ with the intersection points of the top panel. 


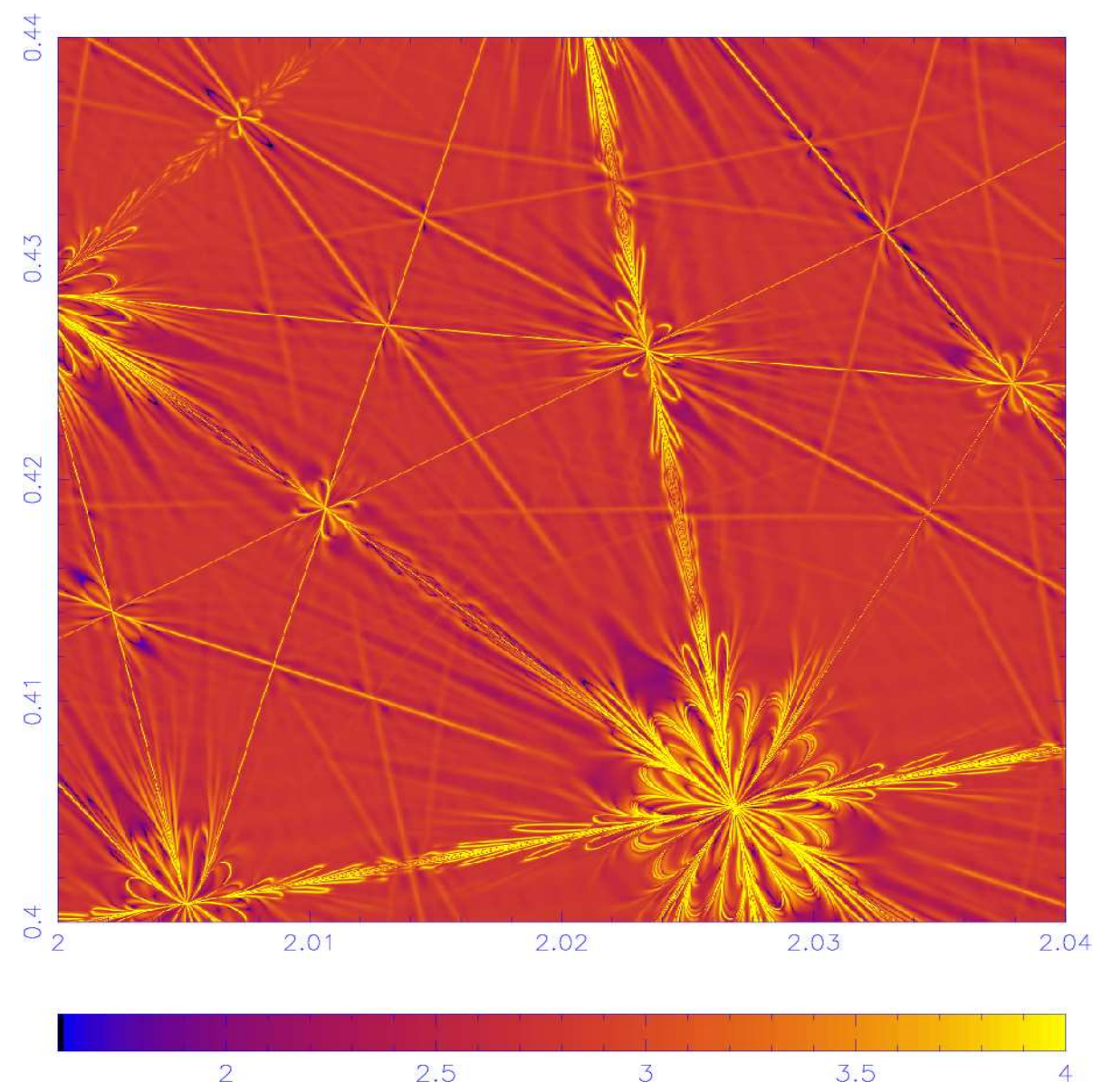

Figure 6: Computation of the FLI for the map (8) with $c=2.1$ and $\varepsilon=0.0001$. On the picture there are many resonance crossings with flower like structures. 

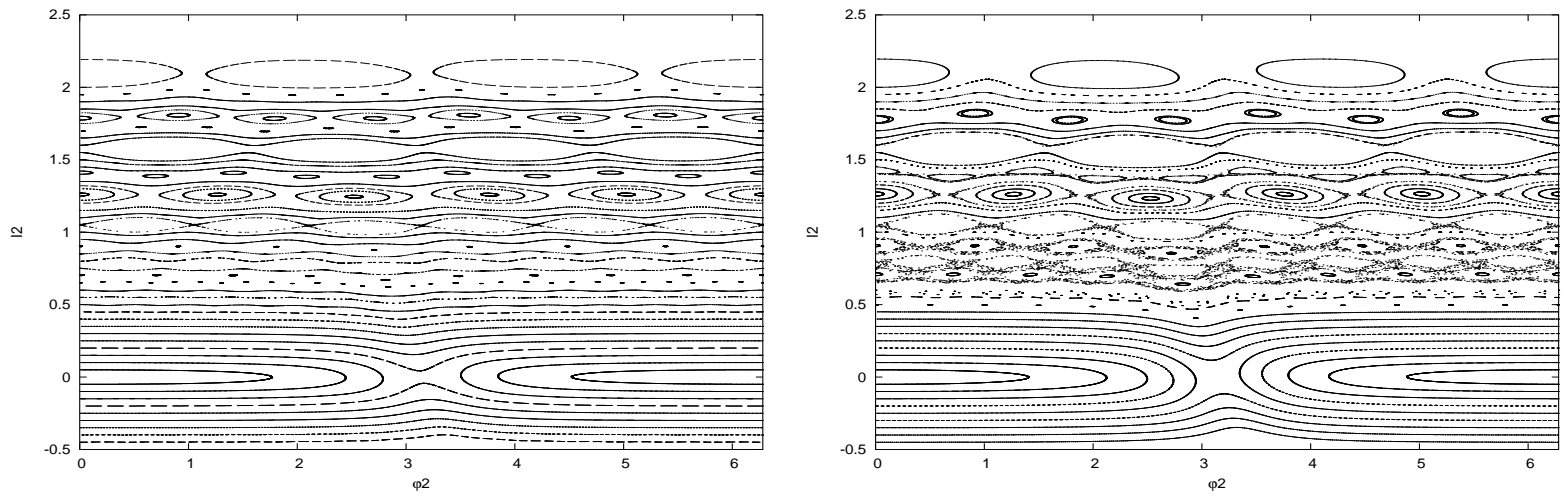

Figure 7: Phase portraits of the restricted map (19) for $\varepsilon=0.002,0.004$ and $c=2.1$. On the right panel we see that the region of the phase space with $I_{2} \in[0.5,1]$ is characterized by the overlapping of resonances. 

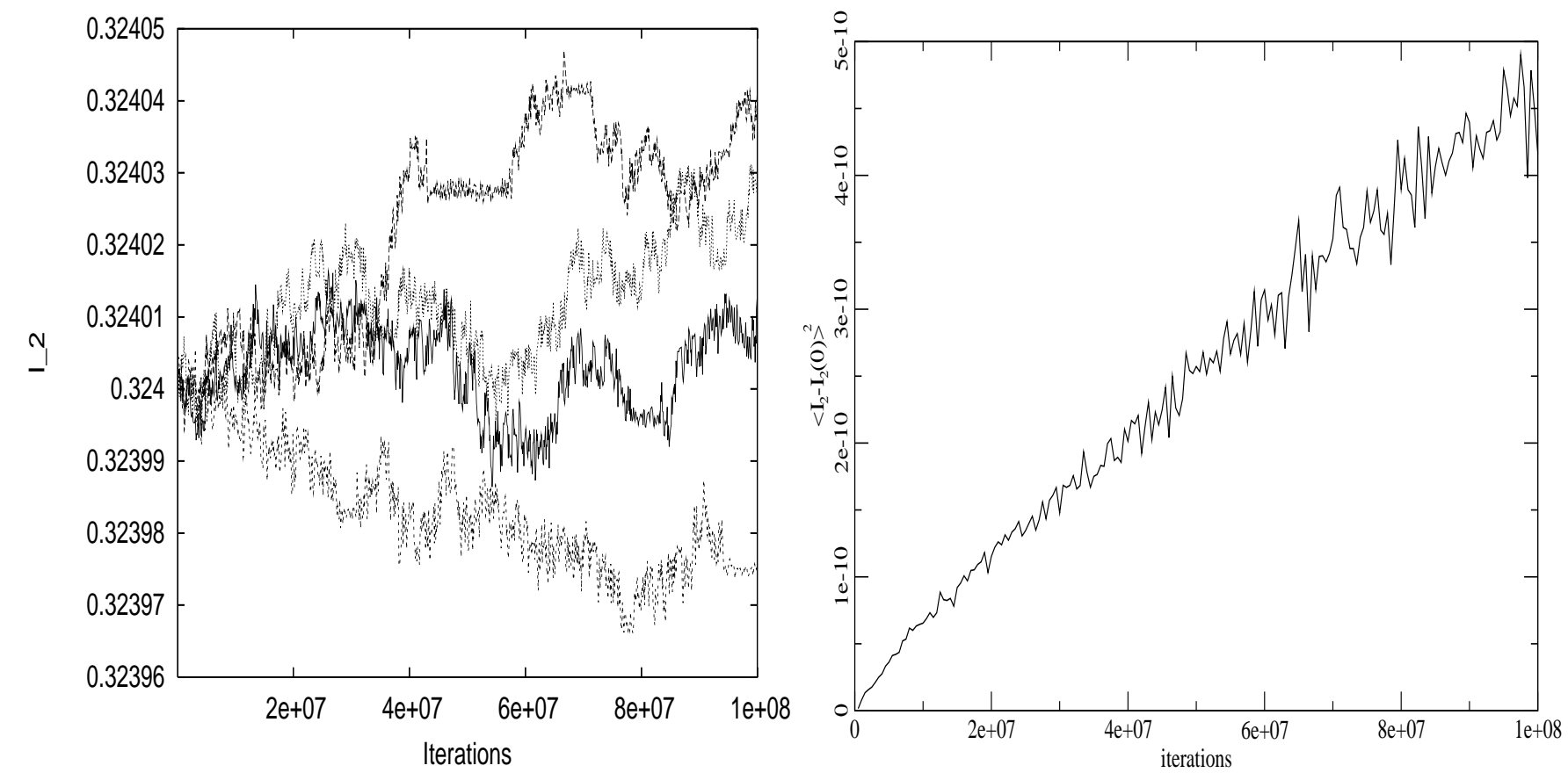

Figure 8: Left panel: Examples of evolution of $I_{2}^{j}(t)$ for orbits with initial conditions suitably close to $\Lambda$. The four initial conditions are: $I_{1}=$ $-10^{-5},-10^{-6}, 10^{-6}, 10^{-5}, I_{2}=0.324, \varphi_{1}=\pi, \varphi_{2}=0$ and $\varepsilon=910^{-5}, c=2.1$. Right panel: Evolution with time of the mean squared distance of a set of $N=1000$ orbits from their initial conditions. The initial conditions are: $I_{1} \in\left[-10^{-5}, 10^{-5}\right]$, $I_{2}=0.324, \varphi_{1}=\pi, \varphi_{2}=0, \varepsilon=910^{-5}$ and $c=2.1$. 


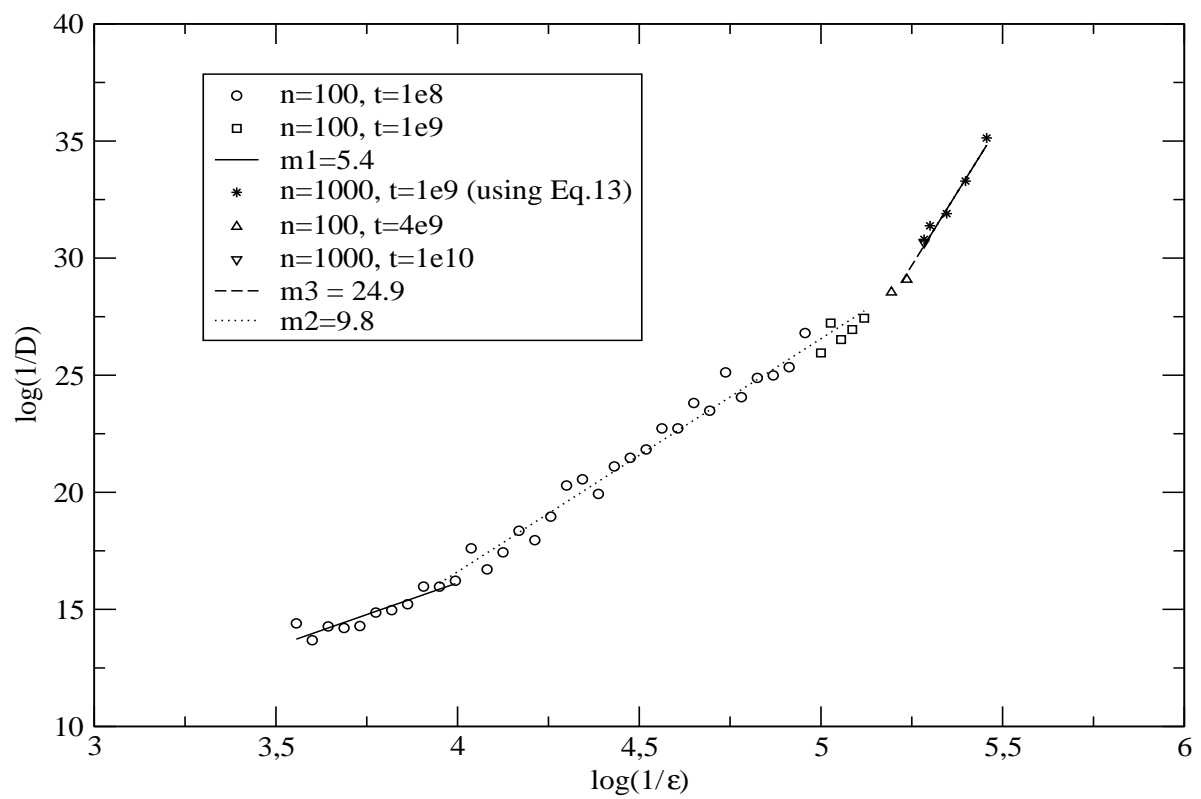

Figure 9: Computation of the diffusion coefficient $D_{2}$ for different values of $\varepsilon$ and $c=2.1$. Depending on the value of $\varepsilon$, we used data sets of $n=100$ or $n=1000$ orbits and total integration times from $t=10^{8}$ up to $t=10^{10}$. The computed diffusion coefficient is well fitted by three different power laws $D_{2}(\varepsilon) \simeq \varepsilon^{m}$ with respectively $m 1=5.4, m 2=9.8$ and $m 3=24.9$ in different intervals of $\varepsilon$. 

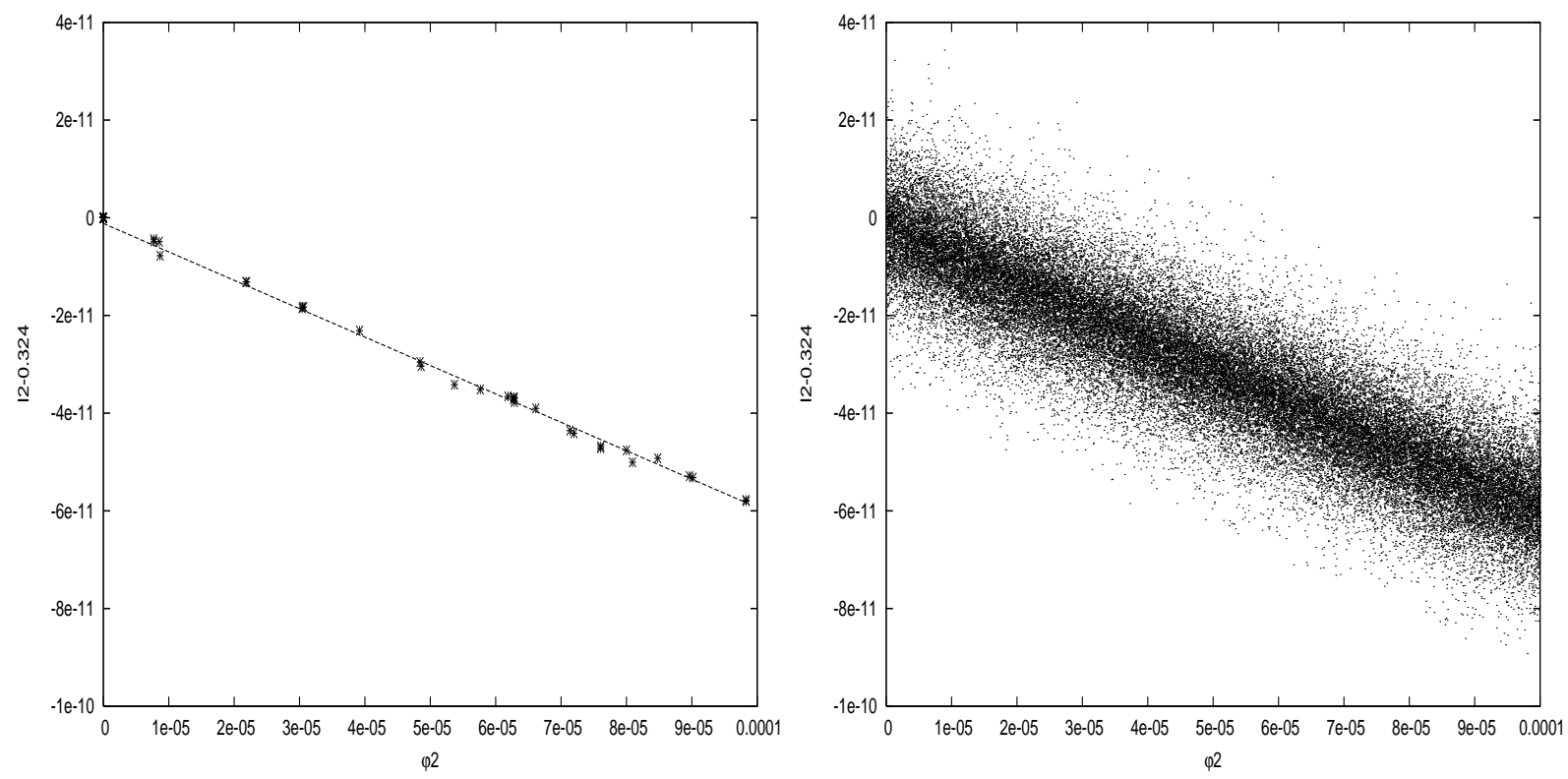

Figure 10: Evolution of $I_{2}^{j}(t)$ versus $\varphi_{2}^{j}(t)$ for a set of $N=1000$ orbits and $t \leq 610^{5}$ (left panel) and $t \leq 10^{9}$ (right panel). The line plotted in the left panel corresponds to the best linear fit $I_{2}=m \varphi_{2}+q$. A diffusion with respect to a direction transverse to this line appears only on the right panel. 